\title{
Effects of coordinated operation management of HVDC links and PSTs on the redispatch in a Central European case study
}

\author{
S. Polster®, H. Renner
}

In this paper, the effects of coordinated operation management of embedded HVDC links and phase shifting transformers on the optimal redispatch costs and volumes are examined. The underlying optimization problem, including these power flow controlling devices, is formulated for linear optimization using DC load flow equations. The evaluation is based on the results of a year-round simulation of the expected energy market and network situation of the ENTSO-E region of the year 2025. The results highlight the need for coordinated operation management of PFCs since uncoordinated operation management increases the accruing redispatch costs.

Keywords: redispatch optimization; coordination of PFCs; linear optimization; operation management of embedded HVDC links; operation management of phase shifting transformers

\section{Auswirkungen einer koordinierten Betriebsführung von HGÜ-Leitungen und PSTs auf den Redispatch in einer zentraleuropäischen Fallstudie.}

In dieser Arbeit werden die Auswirkungen einer koordinierten Betriebsführung von eingebetteten HGÜ-Leitungen und Phasenschiebertransformatoren auf die optimalen Redispatchkosten und -volumen untersucht. Das zugrundeliegende Optimierungsproblem, einschließlich dieser lastflussregelnden Elemente, wird für eine lineare Optimierung unter Verwendung von DC-Lastflussgleichungen formuliert. Die Bewertung basiert auf den Ergebnissen einer Ganzjahressimulation der zu erwartenden Energiemarkt- und Netzsituation der ENTSO-E-Region des Jahres 2025. Die Ergebnisse verdeutlichen die Notwendigkeit einer koordinierten Betriebsführung, da eine unkoordinierte Betriebsführung die anfallenden Redispatchkosten erhöht.

Schlüsselwörter: Redispatchoptimierung; Koordination von lastflussregelnden Elementen; lineare Optimierung; Betriebsführung von eingebetteten HGÜ-Leitungen; Betriebsführung von Phasenschiebertransformatoren

List of Symbols

$\Delta \boldsymbol{\alpha} \quad$ Allowed phase shifter angle change

$\Delta \mathbf{p}_{D C}$ Allowed HVDC power setpoint change

$\Delta \mathbf{p}_{\text {neg }}$ Allowed negative redispatch activation

$\Delta \mathbf{p}_{\text {pos }}$ Allowed positive redispatch activation

A $_{\text {eq }} \quad$ Matrix of equality constraints

$\mathbf{A}_{\text {ineq }}$ Matrix of inequality constraints

$\mathbf{b}_{\text {eq }} \quad$ Vector of equality constraints

$\mathbf{b}_{\text {ineq }}$ Vector of inequality constraints

f Objective function vector

CDC Cost vector of HVDC setpoint change

$\mathbf{c}_{\text {neg }}$ Cost vector of negative redispatch activation

$c_{\text {pos }}$ Cost vector of positive redispatch activation

CPST Cost vector of PST angle change

$c_{O} \quad$ Cost vector of remaining overload

c $U$ Cost vector of solved overload

I Identity matrix

K Diagonal matrix of allowed overload factors

lb Lower bounds of the trial variables

LODF Line outage distribution factor matrix

NDI Node-to-DC incidence matrix

$\mathbf{p}_{\mathrm{L}} \quad$ Vector of active branch powers

$\mathbf{p}_{\mathrm{N}} \quad$ Vector of active node powers
PSDF Phase shifter distribution factor matrix

PTDF Power transfer distribution factor matrix

$\mathbf{S}_{\max }$ Vector of maximal branch power flow

ub Upper bounds of the trial variables

$\mathbf{x} \quad$ Vector of trial variables

$\mathbf{x}^{*} \quad$ Optimum point

$\mathbf{x}_{D C} \quad$ Trial variable vector of HVDC active power setpoint change

$\mathbf{x}_{\text {neg }}$ Trial variable vector of negative redispatch activation

$\mathbf{x}_{\text {pos }}$ Trial variable vector of positive redispatch activation

$\mathbf{x}_{\text {PST }}$ Trial variable vector of PST angle change

$\mathbf{x}_{\bigcirc} \quad$ Trial variable vector of remaining overload

$\mathbf{x}_{\cup} \quad$ Trial variable vector of solved overload

b Arbitrary branch

cc Critical contingency

Csum Overall redispatch costs

dc Number of considered HVDC links

$k_{\mathrm{OL}} \quad$ Utilization factor virtual redispatch

N1 Index indicating $(n-1)$-values

Polster, Stefan, IEAN, Graz University of Technology, Inffeldgasse 18, $8010 \mathrm{Graz}$, Austria (E-mail: stefan.polster@tugraz.at); Renner, Herwig, IEAN, Graz University of Technology, Graz, Austria 
NOL Set of branches not overloaded in the base case

nol Number of branches in NOL

$O L \quad$ Set of branches overloaded in the base case

ol Number of branches in $O L$

pp Number of redispatch power plants

pst Number of considered PST

$V_{\text {sum }}$ Overall redispatch volume

$V_{\text {vir }} \quad$ Virtual redispatch volume

\section{Introduction}

The increasing number of power flow controlling devices (PFC), e.g. phase shifting transformers, converter-based FACTS and embedded HVDC links, in the transmission network allow a more flexible use of the transmission capacity. With the increasing number of PFCs, interfering effects between the devices have to be considered for an optimal utilization of the network capacity. The coordination of the PFC operation becomes thereby a factor not to be dismissed in operational planning as well as in network optimization.

The coordinated control of HVDC links has been already evaluated regarding the capability of reducing the line loadings and applied to a reduced model of the German transmission network in [1, 2]. Further, a coordination of HVDC links to maximize the utilization of renewable energy is discussed in $[3,4]$. The proposed operational strategies of the parallel HVDC links connecting multiple network areas are applied in the North-western Chinese transmission network. The results show, that due to the coordination more renewable energy can be utilized.

The coordination of phase shifting transformers (PST) has been discussed so far regarding the effect on the total transfer capacity between two areas [5], the capabilities of equalizing the loading cross border interconnectors $[6,7]$ in an multi bidding zone network. These studies strongly indicate the need for a coordination between PSTs to avoid additional congestions caused by loop flows.

Additional coordination approaches focusing on security analyses and increasing the interzonal transmission capacities for PSTs and HVDC links are discussed in [8]. However, in this paper no coordination between PSTs and HVDC links are discussed and embedded HVDC links are not considered.

The obvious approach of using optimal power flow calculations to coordinate different types of PFCs, e.g. as discussed in [9], might become computationally complex for large networks leading to impracticable simulation runtimes. To cope with this, either the model's level of detail can be reduced, e.g. by linearizing or decoupling the load flow equations, or the network is separated into different areas affected by the evaluated PFCs as used for example in [10-12].

In [13] control strategies for PSTs and embedded HVDC links for long-term network development planning are evaluated. The focus of this work is set on the reduction of loop flows and thus avoiding overloading of tie lines and reducing unnecessary network extensions. The results indicate, that the coordinated control helps to ease stress introduced on neighbouring networks. Further, the possible relieve of internal tie lines due to optimal exploitation of the net transfer capacity is evaluated.

The effects of coordinated PST and embedded VSC-HVDC link control on decisions considering grid management such as investment, planning, scheduling and operations are discussed in [14]. The results show, that the possibilities of managing loop flows and congestions as well as the additional flexibility allow an operation closer to the system limits.

In contrast to these publications, this paper evaluates the effects on the redispatch costs and volumes of different degrees of PFC coordination with implemented flow-based market coupling FBMC.

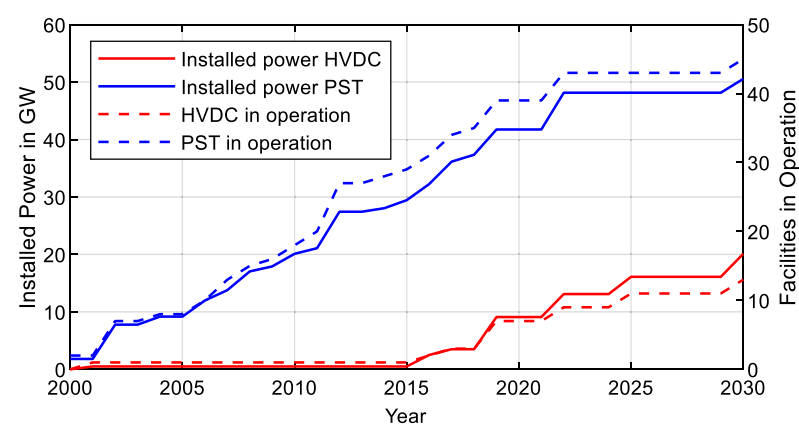

Fig. 1. Installed power of embedded HVDC links / PST and facilities in operation for the ENTSO-E grid

The results presented are based on a full year techno-economic simulation of the ENTSO-E grid of the fictive year 2025. The conducted evaluations are discussed in greater detail in the main author's PhDthesis [15].

The focus on these two types of PFCs is justified by their load flow control capacity, their number, their installed capacity and the availability of data. In Fig. 1 the installed power of embedded HVDC links and PST as well as the number of operational facilities in the ENTSO-E grid are depicted. The data is collected from public sources such as the ten-year development plans.

This paper is organized as follows. In Sect. 2 the optimization problem is formulated. The used linearized DC load flow model of the ENTSO-E grid and the selected critical network elements are described in Sect. 3. The studied scenarios are shown in Sect. 4 and the optimisation results for the scenarios are discussed in Sect. 5 . The conclusions are stated in Sect. 6 .

\section{Formulation of optimization problem}

The optimization of the control setpoints is based on the linearized DC load flow equations. The optimization problem is therefore formulated in a linear form and can be solved efficiently applying simplex algorithms [16]. The optimum $\mathbf{x}^{\star}$ minimizing the costs is found solving the problem specified by

$$
\mathbf{f}^{\top} \cdot \mathbf{x}^{\star}=\min _{\mathbf{x}} \mathbf{f}^{\top} \cdot \mathbf{x} \quad \text { such that }\left\{\begin{array}{c}
\mathbf{A}_{\text {ineq }} \cdot \mathbf{x} \leq \mathbf{b}_{\text {ineq }} \\
\mathbf{A}_{\text {eq }} \cdot \mathbf{x}=\mathbf{b}_{\text {eq }} \\
\mathbf{l} \mathbf{b} \leq \mathbf{x} \leq \mathbf{u b}
\end{array}\right.
$$

In the following, the optimization problem is formulated generally for a combined optimization of redispatch and the control setpoints of embedded HVDCs and PSTs. The symbols are used as given in the List of Symbols above.

The composed vector of trial variables $\mathbf{x}$ consists of the vectors corresponding to positive $\mathbf{x}_{\text {pos }}$ and negative redispatch activation $\mathbf{x}_{n e g}$, the active power setpoint changes of the considered HVDC links $\mathbf{x}_{D C}$ and the angle changes of the considered PSTS $\mathbf{X}_{P S T}$. Further, trial variable vectors $\mathbf{x}_{\cup}$ and $\mathbf{x}_{O}$ are added for branches overloaded in the base case $O L$. The trial variables in $\mathbf{x}_{\cup}$ correspond to the amount of power transfer the formerly overloaded branch is relived under its thermal rating. The trial variables in $\mathbf{x}_{0}$ correspond to the remaining overload on these branches. Their inclusion allows the transfer of the partly linear penalty cost function - zero if the overload is solved and linear for unsolved overloads - into a linear representation. This relaxation in the optimization problem avoids infeasible solutions caused by representation inaccuracies of the underlying model, e.g. missing redispatch power plants and wrong node assignments.

$$
\mathbf{x}^{\top}=\left[\begin{array}{llllll}
\mathbf{x}_{\text {pos }}^{\top} & \mathbf{x}_{\text {neg }}^{\top} & \mathbf{x}_{\mathrm{DC}}^{\top} & \mathbf{x}_{\mathrm{PST}}^{\top} & \mathbf{x}_{\cup}^{\top} & \mathbf{x}_{\mathrm{O}}^{\top}
\end{array}\right]
$$




$$
\begin{aligned}
& \mathbf{x}_{\text {pos }} \in \mathbb{R}^{(p p \times 1)}, \mathbf{x}_{\text {neg }} \in \mathbb{R}^{(p p \times 1)}, \mathbf{x}_{D C} \in \mathbb{R}^{(d c \times 1)} \\
& \mathbf{x}_{\text {PST }} \in \mathbb{R}^{(p s t \times 1)}, \mathbf{x}_{\mathrm{u}} \in \mathbb{R}^{(o / \times 1)}, \mathbf{x}_{\circ} \in \mathbb{R}^{(o / \times 1)}
\end{aligned}
$$

The upper and lower boundaries for all trial variables except $\mathbf{x}_{\cup}$ and $\mathbf{x}_{O}$ are in general defined by the difference of the operation point before optimisation and the corresponding operational limit. To accomplish a strict separation between positive and negative redispatch the lower boundaries of $\mathbf{x}_{\text {pos }}$ are 0 and the upper boundaries of $\mathbf{x}_{\text {neg }}$ are 0 . This separation is necessary, since the costs for positive and negative redispatch may differ even for a single power plant.

The boundaries for $\mathbf{x}_{\cup}$ and $\mathbf{x}_{0}$ are more sophisticated, since they must incorporate the definition of $\mathbf{x}_{\cup}$ as the minimum between the sum of the power flow change and the pre-optimization overload and 0 and the definition of $\mathbf{x}_{\bigcirc}$ as the maximum between the sum of the power flow change and the pre-optimization overload and 0 . Due to these definitions, all elements' values of $\mathbf{x}_{\cup}$ must be negative or 0 and all elements' values of $\mathbf{x}_{0}$ must be positive or 0 . Therefore, the upper boundaries for $\mathbf{x}_{\cup}$ and the lower boundaries for $\mathbf{x}_{\bigcirc}$ are consequently 0 . The lower boundaries of $\mathbf{x}_{\cup}$ of two times the negative thermal rating of the branches $O L$ avoid an overloading in opposite flow direction to the base case. The upper boundaries of $\mathbf{x}_{0}$ define the maximal allowed remaining overload. The matrix $\mathbf{K}$ is a diagonal matrix composed of the allowed overload factors for each branch $O L$.

$$
\begin{aligned}
& \mathbf{l b}^{\top}=\left[\begin{array}{llllll}
\mathbf{0} & \Delta \mathbf{p}_{\text {neg }}^{\top} & \Delta \mathbf{p}_{\mathrm{DC}, \min }^{\top} & \Delta \boldsymbol{\alpha}_{\min }^{\top} & -2 \cdot \mathbf{s}_{\max , O L}^{\top} & \mathbf{0}
\end{array}\right] \\
& \mathbf{u b}^{\top}=\left[\begin{array}{llllll}
\Delta \mathbf{p}_{\text {pos }}^{\top} & \mathbf{0} & \Delta \mathbf{p}_{\mathrm{DC}, \max }^{\top} & \Delta \boldsymbol{\alpha}_{\max }^{\top} & \mathbf{0} & \mathbf{s}_{\max , \mathrm{OL}}^{\top} \cdot \mathbf{K}
\end{array}\right]
\end{aligned}
$$

The dimensions of the sub-vectors forming $\mathbf{l b}$ and $\mathbf{u b}$ equal the dimensions of the corresponding trial variable sub-vectors stated in Eq. (3). These boundaries of $\mathbf{x}_{\cup}$ and $\mathbf{x}_{O}$ ensure that for each branch only one trial variable is not equal to 0 .

The objective function vector $\mathbf{f}$ consists of the cost vectors corresponding to the trial variables. The dimensions of the single vectors equal the dimensions of the corresponding trial variables vectors. All elements in the sub-vector $\mathbf{c}_{\cup}$ are 0 , since they correspond to solved overloads.

$$
\mathbf{f}^{\top}=\left[\begin{array}{llllll}
\mathbf{c}_{\text {pos }}^{\top} & \mathbf{c}_{\text {neg }}^{\top} & \mathbf{c}_{\mathrm{DC}}^{\top} & \mathbf{c}_{\mathrm{PST}}^{\top} & \mathbf{c}_{\cup}^{\top} & \mathbf{c}_{\mathrm{O}}^{\top}
\end{array}\right]
$$

The formulation of the inequality and equality constraints are based on the linearized DC-load flow power transfer distribution factors (PTDF) and phase shifter distribution factors (PSDF) matrices. The constraints must ensure, that the solution is ( $n-1)$-secure. Therefore, the critical (n-1)-PTDFs and -PSDFs as well as the critical $(n-1)$-base case load flow are used. The critical contingencies are determined according to the method described in [15, pp. 43-51]. The following equations show the calculation of the critical (n-1)-PTDFs and (n-1)-PSDFs of branch $b$ and its critical contingency $c c$ as well as the (n-1)-base case load flow using line outage distribution factors (LODF). The PTDFs, PSDFs and LODFs are calculated as discussed in [17-19].

$$
\begin{gathered}
\mathbf{P T D F}_{\mathrm{N} 1, b}=\mathbf{P T D F}_{b}+L O D F_{c c, b} \cdot \mathbf{P T D F}_{c c} \\
\mathbf{P S D F}_{\mathrm{N} 1, b}=\mathbf{P S D F}_{b}+L O D F_{c c, b} \cdot \mathbf{P T D F}_{\mathrm{CC}} \\
p_{\mathrm{L}, \mathrm{N} 1, b}=\mathbf{P T D F}_{\mathrm{N} 1, b} \cdot \mathbf{p}_{\mathrm{N}}
\end{gathered}
$$

Multiplying Eqs. (7) to (9) with the sign of the (n-1)-base case load flows simplifies the formulation of the constraints, since this introduces a sign convention defining the ( $\mathrm{n}$-1)-base case load flows as positive. In the following, ^above a matrix is used to indicate this sign convention.

The effects of the embedded HVDC links are integrated in the optimization problem by multiplying the negative (n-1)-PTDF matrix with the node-to-DC incidence matrix NDI. The NDI matrix maps the terminals of the embedded HVDC links to the nodes of the network.

The inequality constraints - Eqs. (10) and (11) - are only defined for the branches not overloaded in the base case NOL. Their formulation must ensure, that these branches are still not overloaded after the optimization. Therefore, the cumulated effects of the activated redispatch and PFC control are only allowed to increase the branch flow with the difference between its thermal rating and the (n-1)base case load flow. The entries in the matrix $\mathbf{A}_{\text {ineq }}$ corresponding to $\mathbf{x}_{\cup}$ and $\mathbf{x}_{0}$ are all 0 .

The equality constraints defined by the matrix $\mathbf{A}_{\text {eq }}$ and the vector $\mathbf{b}_{\text {eq }}$ - Eqs. (12) and (13) - consist of the balance of the activated positive and negative redispatch power. Further the balance of the cumulated effects of the activated redispatch, PFC control and the negative values of $\mathbf{x}_{\cup}$ and $\mathbf{x}_{O}$ with the base case overload has to be considered for the branches $O L$.

$$
\begin{aligned}
& \mathbf{A}_{\text {ineq }}=[\underbrace{\widehat{\operatorname{PTDF}}_{\mathrm{N} 1, N O L, R D}}_{(n o l \times p p)} \underbrace{\widehat{\operatorname{PTDF}}_{\mathrm{N} 1, N O L, R D}}_{(n o l \times p p)} \underbrace{-\widehat{\mathbf{P T D F}}_{\mathrm{N} 1, N O L} \cdot \mathbf{N D I}}_{(n o l \times d c)} \underbrace{\widehat{\operatorname{PSDF}}_{\mathrm{N} 1, N O L}}_{(n o l \times p p)} \underbrace{\mathbf{0}}_{(n o l \times o l)} \underbrace{\mathbf{0}}_{(n o / \times o l)}] \\
& \mathbf{b}_{\text {ineq }}=\mathbf{s}_{\max , N O L}-\left|\mathbf{p}_{\mathrm{L}, \mathrm{N} 1, \mathrm{NOL}}\right|
\end{aligned}
$$

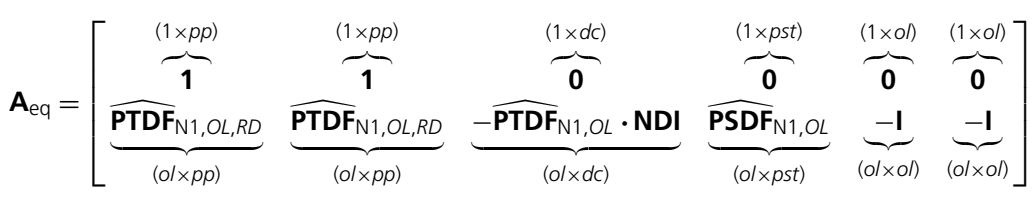

$$
\begin{aligned}
& \mathbf{b}_{\mathrm{eq}}=\left[\begin{array}{c}
\mathbf{0} \\
\mathbf{s}_{\mathrm{max}, \mathrm{OL}}-\left|\mathbf{p}_{\mathrm{L}, \mathrm{N} 1, \mathrm{OL}}\right|
\end{array}\right]
\end{aligned}
$$

The following simple example shall illustrate the behaviour of $\mathbf{x} \cup$ and $\mathbf{x}_{O}$ and the corresponding constraints and costs. Assume two branches $i$ and $j$, which are overloaded with 0.5 pu and $1.5 \mathrm{pu}$ in the base case. Consequently, they are added to the branches $O L$ and corresponding elements. The corresponding elements in the vector $\tilde{\mathbf{b}}_{\text {eq }}$ are $-0.5 \mathrm{pu}$ and $-1.5 \mathrm{pu}$. The power changes caused by the optimal solution for redispatch, HVDC links and PSTs are $-0.8 \mathrm{pu}$ for branch $i$ and -1 pu for branch $j$. To comply with the equality 
constraints the elements corresponding to $i$ and $j$ in $\mathbf{x}_{\cup}$ and $\mathbf{x}_{0}$ are nonzero. Focusing first on branch $i$, the sum of the power change and initial overload is $-0.3 \mathrm{pu}$. Since this value is smaller than 0 , the value of the corresponding elements in $\mathbf{x}_{\cup}$ becomes $-0.3 \mathrm{pu}$ and in $\mathbf{x}_{0} 0$ due to its lower limit. The sum of power change and initial overload for branch $j$ is $0.5 \mathrm{pu}$. Since this value is higher than 0 , the value of the corresponding elements in $\mathbf{x}_{\cup}$ becomes 0 due to its upper limit and the value in $\mathbf{x}_{\bigcirc} 0.5 \mathrm{pu}$. The costs corresponding to $\mathbf{x}_{\cup}$ are all 0 , therefore only the remaining overload equalling the values in $\mathbf{x}_{0}$ increases the objective function value

To maintain a simple ( $n-1)$-security condition for parallel HVDClinks it must be ensured, that in case of an HVDC link outage the power transfer of this link can be shifted to the parallel HVDC links. Possible AC grid overloading in case of an HVDC outage are thereby not considered. This condition is implemented by adding an inequality constraint limiting the sum of the power transfer via the parallel HVDC-links to the sum of their nominal powers minus the nominal power of the strongest parallel HVDC link.

The resulting redispatch costs of the optimal solution $\mathbf{x}^{*}$ are composed of costs of the activated positive and negative redispatch and the costs of a virtual redispatch corresponding to the remaining overload. The cost of the virtual redispatch is calculated as the mean value of the positive redispatch costs. The virtual redispatch's volume is calculated from the sum of the remaining overloads weighted with a utilization factor $k_{\mathrm{OL}}$. $k_{\mathrm{OL}}$ gives the efficiency of the virtual redispatch. E.g. a value of 0.5 means, that the virtual redispatch has to be twice the amount of the remaining overload to solve it. To avoid an overestimation of the virtual redispatch, remaining overloads occurring in series to each other - e.g. lines split up in sections without load/generation in between - are considered only ones.

$$
\begin{gathered}
V_{\text {vir }}=\frac{1}{k_{\mathrm{OL}}} \cdot \sum \mathbf{x}_{\mathrm{O}}^{*} \\
k_{\mathrm{OL}}=(0,1] \\
V_{\text {sum }}=\sum \mathbf{x}_{\text {pos }}^{*}+V_{\text {vir }} \\
C_{\text {sum }}=\mathbf{c}_{\text {pos }}^{\top} \cdot \mathbf{x}_{\text {pos }}^{*}+\mathbf{c}_{\text {neg }}^{\top} \cdot \mathbf{x}_{\text {neg }}^{*}+\overline{\mathbf{c}_{\text {pos }}} \cdot V_{\text {vir }}
\end{gathered}
$$

\section{Model description}

The network model represents the 220-kV- and 380-kV-grid of the ENTSO-E region on nodal basis. Power transfers with Great Britain and other grid areas connected asynchronously via HVDC links - are considered as power injections at the corresponding HVDC terminals. Subordinate grid levels are in general substituted as residual generation and load, whereas parts of the 110-kV-grid essential for international power flows are modelled in detail.

The focus of this work is set on an assumed FBMC covering the bidding zones of Austria, the Benelux countries, Czech Republic, France, Germany, North-Italy, Poland and Switzerland. The other bidding zones of the ENTSO-E region and the connected energy markets of Great Britain, Ireland and Scandinavia are included in the market model in accordance with their expected net transfer capacities of the year 2025

The branches of the considered network are plotted in Fig. 2. Three network areas are distinguished depending on the generation's level of detail, redispatch capacity modelling and integration in the FBMC. Area 1 - plotted solid red - corresponds to the FBMC region and its generation and redispatch are modelled on power plant basis with accurate power limitations. The generation and redispatch of Area 2 - plotted dotted green - are modelled the same

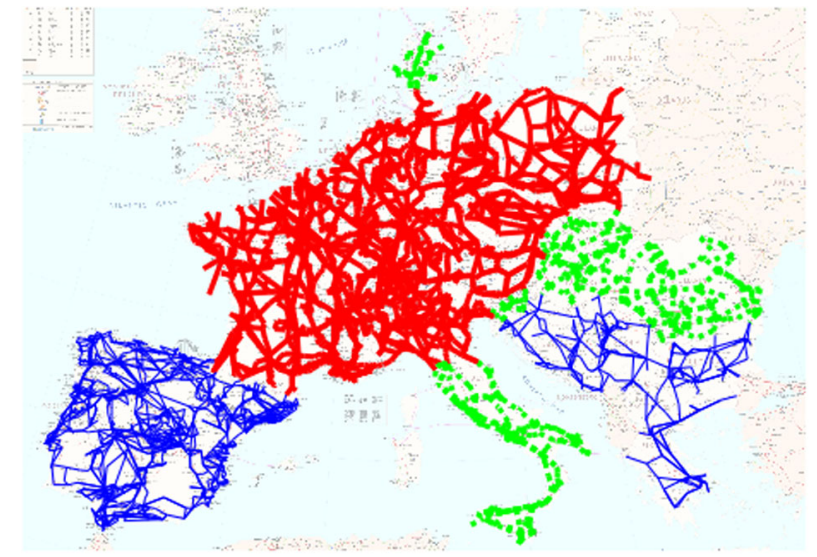

Fig. 2. Modelled grid areas: solid red Area 1, dotted green Area 2 and thin solid blue Area 3 (Color figure online)

Table 1. Elements in included in the separated grid areas

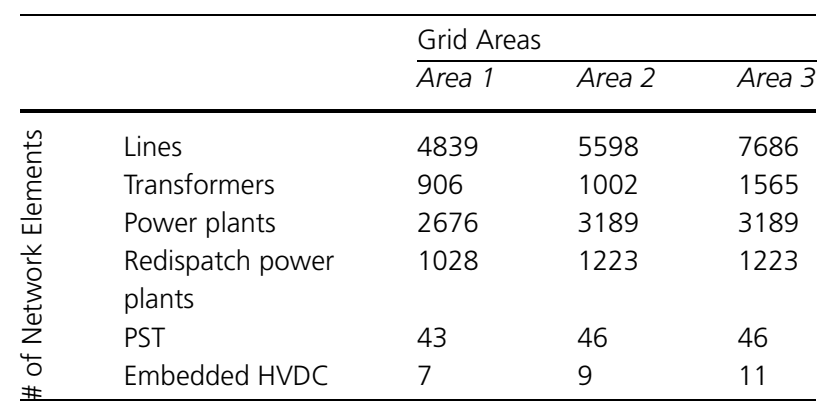

as in Area 1 and its market model is based on net transfer capacities. Area 3 -plotted thin solid blue - generation is modelled by scaling the residual bidding zones' generation of the market result with the static generation data of the basic ENTSO-E model and no redispatch is considered in these bidding zones. The loads in all three areas are implemented by scaling the residual load of the single bidding zones with the static load data of the basic ENTSO-E model. A breakdown of the elements included in the electrical model and single grid areas is given in Table 1 .

The costs of a redispatch action are based on variable costs - e.g. fuel of thermal power plants - and opportunity costs, if the primary energy source is limited - e.g. for hydro storage power plants. However, start-up costs and minimal active power limits are not considered. Any change of the control setpoints of the included embedded HVDC links and PSTs are assumed as costless. Details of the market simulation and the determination of redispatch action costs of the individual power plants are not further discussed, since these tasks were performed on an external model. Figure 3 shows the work flow and demarcation between the author's work and externally contributed data and models.

\section{Studied scenarios}

To highlight the effects of a combined optimization of price driven redispatch, and control setpoint changes of embedded HVDC links and PSTs the following seven scenarios are compared. The costs of redispatch actions and the base case load flow are the same for all scenarios. The objective of the optimization is to minimise the overall redispatch costs on an hourly base in the fictive year 2025.

1. Reference Scenario The model corresponds to the expected grid situation in 2025 including the realistic AC grid expansion 


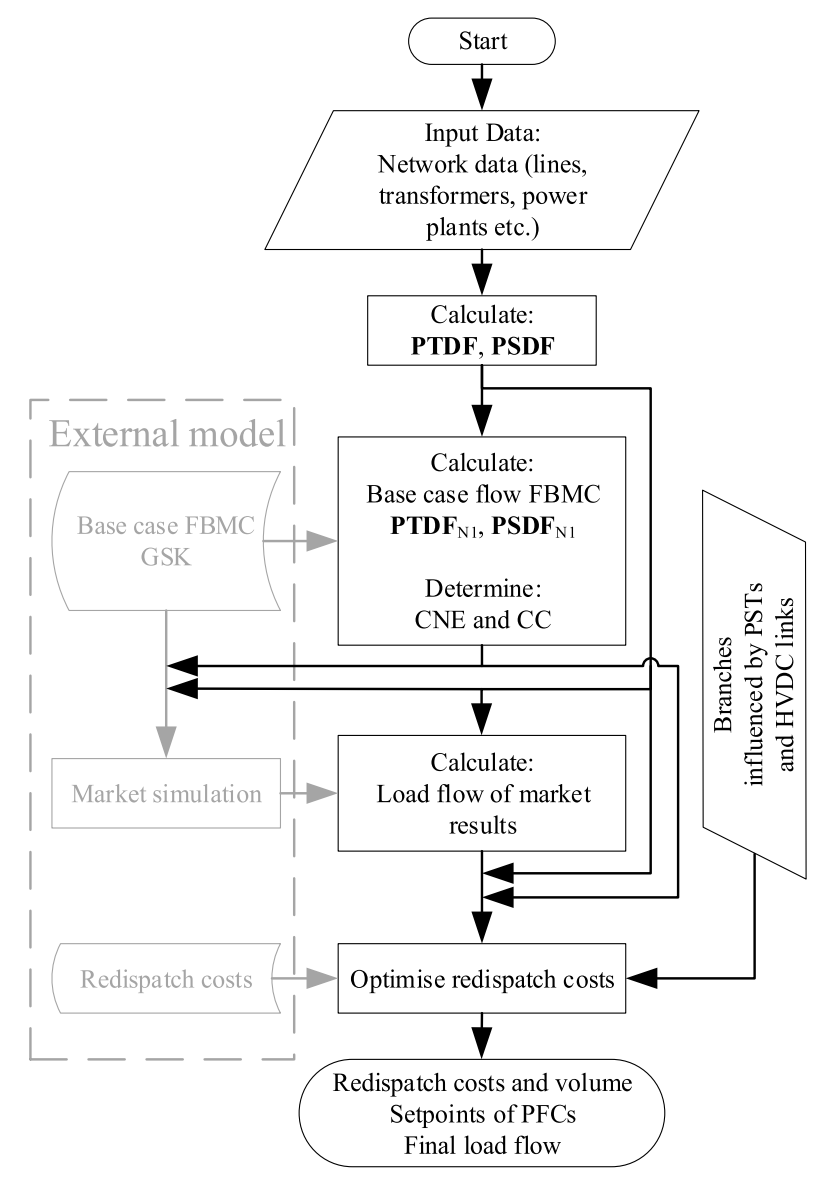

Fig. 3. Work flow of the simulations. Black are the authors' own contribution, grey are the work steps contributed externally

projects finished until 2025. In this scenario PSTs are treated as normal transformers without power flow controlling function and the embedded HVDC links are out of operation. The redispatch is optimised as described in Sect. 2.

2. Uncoordinated PST and redispatch optimization The model basic data and branches considered for the redispatch optimization are unchanged to the reference scenario. Additionally, the PST setpoints are optimized separately before the redispatch optimization. The setpoints are determined as the minimal phase shifter angle necessary for (n-1)-security of the branches influenced by the PSTs. These branches of interest $(\mathrm{BOI})$ are all branches of Area 2 , which loading can be changed by more than 0.3 pu by a single PST's range of operation. The resulting power flow after PST optimization is used as input for the redispatch optimization. The embedded HVDC links are still out of operation.

3. Uncoordinated HVDC link and redispatch optimization The model basic data and branches considered for the redispatch optimization are unchanged to the reference scenario. The HVDC link setpoints are derived by minimizing the loading of the branches affected by the considered HVDC links under ( $n-1)$ secure AC network conditions. The thereby considered $\mathrm{BOI}$ are all branches of Area 2 with a change in loading of more than 0.1 pu caused by one single HVDC link's range of operation. The (n-1)-security condition for HVDC links is implemented for the parallel HVDC links in Germany and across the Mediterranean Sea. The PST are treated as normal transformers without power flow controlling function. The resulting power flow after HVDC link optimization is used as input for the redispatch optimization.

4. Uncoordinated HVDC link, PST and redispatch optimization The model basic data and branches considered for the redispatch optimization are unchanged to the reference scenario. The HVDC link setpoints are derived as in scenario 3. The PST setpoints are determined as in scenario 2, but with the power flow after HVDC link optimization as input. The resulting power flow after PST optimization is used as input for the redispatch optimization.

5. Coordinated PST and redispatch optimization The model basic data and branches considered for the redispatch optimization are unchanged to the reference scenario. The optimization of the PST setpoints and redispatch activation is executed in parallel as described in Sect. 2. Since the embedded HVDC links are out of operation, the results of this scenario highlight the effect of coordinated PST optimization on the necessary redispatch volume.

6. Coordinated HVDC link and redispatch optimization The model basic data and branches considered for the redispatch optimization are unchanged to the reference scenario. The optimization of the HVDC setpoints and redispatch activation is executed in parallel as described in Sect. 2. The simplified (n-1)-security condition for the parallel HVDC links is implemented additionally. In this scenario the effect of the considered embedded HVDC links on the necessary redispatch are highlighted. The PSTs are treated as normal transformers without power flow controlling function.

7. Coordinated HVDC link, PST and redispatch optimization The model basic data and branches considered for the redispatch optimization are unchanged to the reference scenario. The optimization is executed as described in Sect. 2 and includes the PST and HVDC link setpoints as well as the redispatch. As in all other scenarios including HVDC links, the simplified (n-1)-security condition for the parallel HVDC links in Germany and across the Mediterranean Sea is implemented.

The redispatch costs and volumes for these seven scenarios are evaluated for the critical network elements (CNE) defined for the FBMC according to the regulatory stated by ACER. The geographical distribution of the CNE branches as well as the branches determined for the uncoordinated optimization of PST and HVDC link setpoints are depicted in Fig. 4.

The optimization is executed for all hours of the fictive year 2025, whereby the following four seasons are implemented in the market simulation with different generation shift keys and load patterns:

- Off-peak Winter - Season 1

1st of October - 31st of March, workday 22:00 to 10:00 and weekends

- Off-peak Summer - Season 2

1st of April - 30th of September, workday 22:00 to 10:00 and weekends

- Peak Winter - Season 3

1st of October - 31st of March, workday 10:00 to 22:00

- Peak Summer - Season 4

1st of April - 30th of September, workday 10:00 to 22:00

\section{Results}

The results of scenario 1 are discussed first, since they are used as reference to highlight the effects of the different degree of coordination of the PFCs. The following results are based on a utilization factor of 0.5 . The values given are referenced to the mean value of the hourly redispatch costs respectively hourly redispatch volumes.

The seasonal distribution of the hourly redispatch costs of scenario 1 grouped to the seasons are depicted as boxplots in the left 

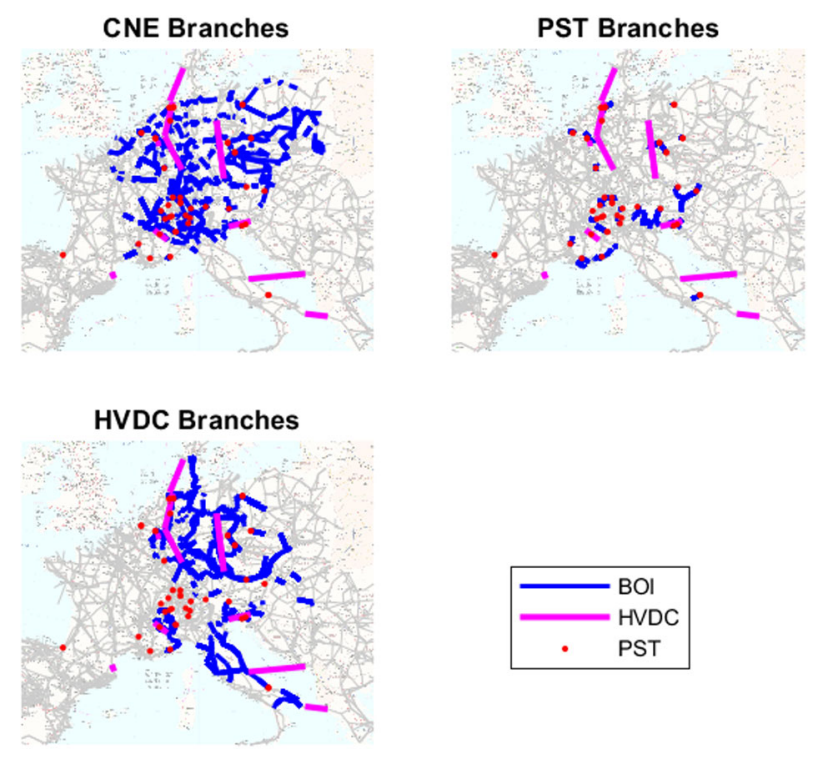

Fig. 4. Geographical distribution of considered branches. CNE branches and branches for the uncoordinated optimization of PSTs and HVDCs
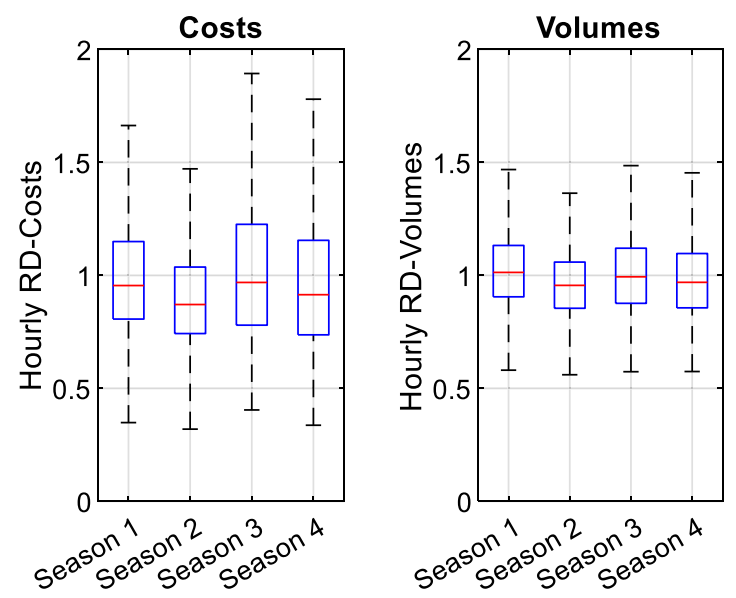

Fig. 5. Distribution of hourly redispatch costs and volumes of scenario 1 . The left graph shows the hourly costs, the right graph the hourly volumes

graph of Fig. 5. The results show the expected behaviour with higher redispatch costs in the winter season and during peak hours in the distribution and mean values.

The distribution of the hourly redispatch volumes is depicted in the right graph of Fig. 5. As expected, the necessary redispatch volumes in the winter seasons are higher than for the summer seasons. However, it can be seen, that the smaller volume changes between the seasons trigger higher cost changes. This highlights the nonlinearity of the redispatch costs to the redispatch volume. The higher increase in the costs is caused by activation of more expensive and less effective redispatch power plants as soon as better located redispatch power plants reach their limitation.

The mean values of the hourly redispatch costs and volumes are given in Table 2.

The results of the scenarios 2 to 7 are normalized to scenario 1 . This means, that the results are divided by the corresponding result
Table 2. Hourly mean costs and volumes of scenario 1

\begin{tabular}{lll}
\hline & RD-Costs & RD-Volumes \\
\hline Season 1 & 1.02 & 1.02 \\
Season 2 & 0.94 & 0.97 \\
Season 3 & 1.06 & 1.01 \\
Season 4 & 1.01 & 1.00 \\
\hline
\end{tabular}

of scenario 1 for each hour. Values above 1 pu indicate an increase and values below 1 pu a reduction. The thereby normalized redispatch costs are depicted in the left graph of Fig. 6 . The boxplots in the figure are grouped according to the seasons and the single scenarios are marked by the box colours.

An increase of redispatch costs for scenario 2 and 4 is noticeable. Since no general increase occurs for scenario 3 , it can be concluded, that the increasing redispatch costs are caused by the PSTs. This intuitively not expected increase is explained by the considered PSTs reducing power flows on their corresponding $\mathrm{BO}$ and thereby increasing power flows on the other branches in the network. The relief of the $\mathrm{BO}$ leads to more overloaded and higher overloaded branches in the surrounding network causing additional redispatch forcing the optimizer to use more expensive and less effective redispatch power plants. This effect is further pronounced due the focus on the CNE branches, since not all PST'S BOI are CNE branches, which cause a higher loading on branches considered in the redispatch optimization without the advantage of less loaded branches.

The comparison between scenario 5 and 6 shows, that the inclusion of PSTs in a coordinated redispatch optimization leads to a higher cost reduction than the inclusion of HVDC links. This is explained on one side by the distribution of PSTs over a larger network area and on the other side on their location, since PSTs are in general equipped to branches who caused massive power flow restrictions and redispatch costs in the past.

The lowest redispatch costs are obtained as expected in scenario 7 with a complete coordination between PSTs, HVDC links and redispatch activation. It is noticeable, that negative redispatch costs appear as can be seen by the whiskers of the corresponding boxplots in Fig. 6 . This is caused by enabling a substitution of thermal power generation with cheaper sources due to additional transmission capacity.

The redispatch volumes normalized to scenario 1 is given in the right graph of Fig. 6 . The volumes increase for scenario 2 and 4 and decrease for the other scenarios, which is in accordance with the change of the redispatch costs discussed above. However, the volume changes are smaller due to the nonlinear relationship between redispatch volume and costs.

Comparing the redispatch volumes and costs of scenario 5 and 7 to each other, it is observable, that in scenario 7 less costs are achieved with the use of similar redispatch volumes. This shows that the coordination of PSTs and HVDC links enables the use of cheaper redispatch power plants due to effective and efficient redistribution of the power flow.

Table 3 summarizes the normalized optimization results of the scenarios 2 to 7 by the mean values of the hourly results.

The share of the virtual redispatch in the overall redispatch costs and volumes for the seven scenarios is given in Table 4 . As can be seen by the small values, the introduction of the virtual redispatch has no significant effect on the optimization result.

\section{Conclusion}

In this paper the effects of coordinating the operation points of PFCS to achieve $(n-1)$-secure operation of the system with a cost efficient 

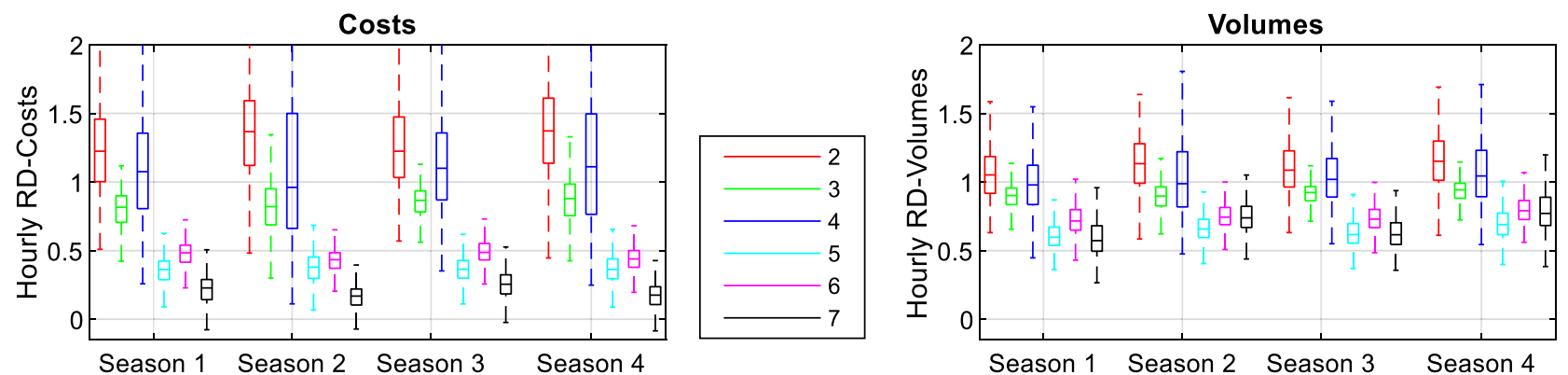

Fig. 6. Hourly redispatch costs and volumes of the scenarios 2 to 7 normalized to scenario 1 . The results are grouped by season and the scenarios are indicated by the box colours (Color figure online)

Table 3. Mean values of the normalized hourly redispatch costs and volumes

\begin{tabular}{lll}
\hline & $\begin{array}{l}\text { Hourly RD-Costs } \\
\text { referred to Scenario 1 }\end{array}$ & $\begin{array}{l}\text { Hourly RD-Volumes } \\
\text { referred to Scenario 1 }\end{array}$ \\
\hline Scenario 2 & 1.31 & 1.11 \\
Scenario 3 & 0.84 & 0.91 \\
Scenario 4 & 1.12 & 1.03 \\
Scenario 5 & 0.37 & 0.65 \\
Scenario 6 & 0.45 & 0.76 \\
Scenario 7 & 0.19 & 0.70 \\
\hline
\end{tabular}

Table 4. Share of the virtual redispatch in the overall redispatch costs and volumes

\begin{tabular}{lll}
\hline & $\begin{array}{l}\text { Virtual RD-Costs } \\
\text { in \% }\end{array}$ & $\begin{array}{l}\text { Virtual } \\
\text { RD-Volumes in \% }\end{array}$ \\
\hline Scenario 1 & 1.42 & 0.70 \\
Scenario 2 & 1.65 & 0.97 \\
Scenario 3 & 1.45 & 0.66 \\
Scenario 4 & 2.87 & 1.55 \\
Scenario 5 & 1.40 & 0.43 \\
Scenario 6 & 1.33 & 0.40 \\
Scenario 7 & 0.64 & 0.09 \\
\hline
\end{tabular}

redispatch are evaluated. Therefore, it is assumed, that the redispatch measures are coordinated between the network operators of the considered network area. The redispatch is activated based on the optimization of its costs, whereby depending on the scenario PFCs are either completely excluded for the base case scenario 1, controlled without coordination to the redispatch activation for scenarios 2 to 4 or included into the optimization of the redispatch for the scenarios 5 to 7 .

The resulting redispatch costs and volumes allow the following conclusions:

Uncoordinated control of PSTs (scenario 2) leads to an increase of the redispatch costs. This is explained by the combination of a local focus relieving the PST equipped branches at the costs of causing and/or increasing overloads in the rest of the network and the nonlinearity of redispatch costs. Further, PSTs are mainly located on tie lines and bottle necks between network zones. In general, more options to relieve these branches with redispatch exist than for branches in more meshed network areas. Therefore, the PSTS reliving these branches force the redispatch optimizer to use less efficient redispatch power plants. The cost and volume increasing effect occurs also in scenario 4.

Uncoordinated control of HVDC links (scenario 3) decreases the necessary redispatch volume and costs in the majority of load situations. The decrease is reasonable since the HVDC links offer additional power transfer capacity. The same effects and also occurs in scenario 4

Comparing the effects of coordinated control of PSTs and HVDC links separately (scenarios 5 and 6) shows, that PSTs are more effective in the reduction of necessary redispatch volume and costs. This is caused by their location on historical identified network bottle necks and the high local power flow sensitivity. The coordination with the redispatch activation prevents unfavourable PST setpoints causing additional overloads.

The combined coordination PSTs and HVDC links leads expectantly to the highest decrease in redispatch costs. However, the activated redispatch volume is only reduced comparably to scenario 5 . This highlights that the optimizer sets the operation points of the PSTs and HVDC links to allow the use of cheapest redispatch power plants and not the most efficient ones.

The results allow a qualitative assessment of the scenarios based on critical network elements. However, it must be kept in mind, that cost and volume decreasing respectively increasing effects of PFCs are overestimated, if not all branches are considered for the optimization.

Funding Note Open access funding provided by Graz University of Technology.

Publisher's Note Springer Nature remains neutral with regard to jurisdictional claims in published maps and institutional affiliations.

Open Access Dieser Artikel wird unter der Creative Commons Namensnennung 4.0 International Lizenz veröffentlicht, welche die Nutzung, Vervielfältigung, Bearbeitung, Verbreitung und Wiedergabe in jeglichem Medium und Format erlaubt, sofern Sie den/die ursprünglichen Autor(en) und die Quelle ordnungsgemäß nennen, einen Link zur Creative Commons Lizenz beifügen und angeben, ob Änderungen vorgenommen wurden. Die in diesem Artikel enthaltenen Bilder und sonstiges Drittmaterial unterliegen ebenfalls der genannten Creative Commons Lizenz, sofern sich aus der Abbildungslegende nichts anderes ergibt. Sofern das betreffende Material nicht unter der genannten Creative Commons Lizenz steht und die betreffende Handlung nicht nach gesetzlichen Vorschriften erlaubt ist, ist für die oben aufgeführten Weiterverwendungen des Materials die Einwilligung des jeweiligen Rechteinhabers einzuholen. Weitere Details zur Lizenz entnehmen Sie bitte der Lizenzinformation auf http://creativecommons.org/licenses/by/4.0/ deed.de. 


\section{References}

1. Frey, K., Wiest, P., Rudion, K., Christian, J. (2016): Automated operation of paralle VSC HVDC links within an interconnected AC network. In IEEE power energy soc. gen. meet. (Vol. 2016, pp. 1-5). https://doi.org/10.1109/PESGM.2016.7741348.

2. Frey, K., Rudion, K., Christian, J. (2016): Automated operation approach for embedded HVDC links during ( $\mathrm{N}-1)$-conditions in the AC system. In 2016 IEEE int. energy conf. ENERGYCON 2016 (pp. 1-6). https://doi.org/10.1109/ENERGYCON.2016.7514117.

3. Zhang, H., Zhang, S. (2018): A new strategy of HVDC operation for maximizing renewable energy accommodation. In IEEE power energy soc. gen. meet. (Vol. 2018, pp. 1-6). https://doi.org/10.1109/PESGM.2017.8274477.

4. Xu, F., Xie, X., Shi, L., Yu, L. (2019): A new operation model of HVDC tie-line for promoting renewable energy accommodation. In Proc. - IEEE int. conf. energy internet, ICEI 2019 (pp. 290-297). https://doi.org/10.1109/ICEI.2019.00058

5. Verboomen, J. (2008): Optimisation of transmission systems by use of phase shifting transformers. Delft: TU Delft.

6. Korab, R., Owczarek, R. (2016): Impact of phase shifting transformers on cross-borde power flows in the Central and Eastern Europe region. Bull. Pol. Acad. Sci., Tech. Sci., 64(1), 127-133. https://doi.org/10.1515/bpasts-2016-0014.

7. Verboomen, J., Van Hertem, D., Schavemaker, P. H., Kling, W. L., Belmans, R. (2007): Border-flow control by means of phase shifting transformers. In 2007 IEEE Lausanne POWERTECH, proc. (pp. 1338-1343). https://doi.org/10.1109/PCT.2007.4538510.

8. Van Hertem, D., Eriksson, R., Söder, L., Ghandhari, M. (2010): Coordination of multiple power flow controlling devices in transmission systems. In IET conf. publ., 2010 (Vo. 570 (P). https://doi.org/10.1049/cp.2010.0961.

9. Glanzmann, G., Andersson, G. (2005): Coordinated control of FACTS devices based on optimal power flow. In Proc. 37th annu. north am. power symp. 2005 (Vol. 2005, pp. 141-148). https://doi.org/10.1109/NAPS.2005.1560515.

\section{Authors}

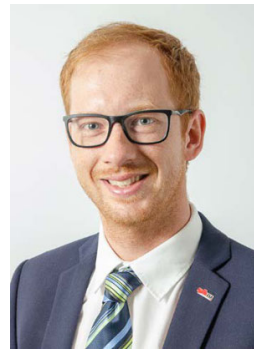

\section{Stefan Polster}

received the master degree in 2016 and the Ph.D. degree in 2021 in electrical engineering, both from Graz University of Technology, Austria. His research mainly deals with power system dynamics and operation. In 2017 he became a recipient of a DOC Fellowship of the Austrian Academy of Sciences at the Institute of Electrical Power Systems, Graz University of Technology.
10. Hug-Glanzmann, G., Andersson, G. (2009): N-1 security in optimal power flow control applied to limited areas. IET Gener. Transm. Distrib., 3(2), 206-215. https://doi. org/10.1049/iet-gtd:20080112.

11. Hug-Glanzmann, G., Andersson, G. (2007): Coordinated control of FACTS devices in power systems for security enhancement. In 2007 iREP symp. bulk power syst. dyn. control - VII, revital. oper. reliab. https://doi.org/10.1109/IREP.2007.4410510.

12. Hug, G. (2013): Distributed optimization to enable a flexible power grid with corrective power flow control. In IEEE power energy soc. gen. meet. https://doi.org/10.1109/ PESMG.2013.6672482.

13. Bauer, R., Weidner, J., Salehinajafabadi, S. (2015): Control strategies of phase-shifting transformers in long-term network development. In Int. ETG congr. (pp. 356-362), no. Figure 2

14. Van Hertem, D., Rimez, J., Belmans, R. (2013): Power flow controlling devices as a smart and independent grid investment for flexible grid operations: Belgian case study. IEEE Trans. Smart Grid, 4(3), 1656-1664. https://doi.org/10.1109/TSG.2013. 2249597

15. Polster, S. (2021): Enhancing secure grid operation by using PFCs and WAMS. Graz: Graz University of Technology.

16. Chychykina, I., Wolter, C. K. M. (2017): Comparison of different redispatch optimization strategies. In 2017 IEEE Manchester PowerTech, Powertech 2017 (Vol. 3). https:// doi.org/10.1109/PTC.2017.7981077.

17. Van Den Bergh, K., Delarue, E., William, D. (2014): DC power flow in unit commitment models (no. May).

18. Guo, J., Fu, Y., Li, Z., Shahidehpour, M. (2009): Direct calculation of line outage distribution factors. IEEE Trans. Power Syst., 24(3), 1633-1634. https://doi.org/10.1109/ TPWRS.2009.2023273.

19. Polster, S., Renner, H. (2018): Generalisation of the Line Outage Distribtution Factors on Phase Shifting Transformers.

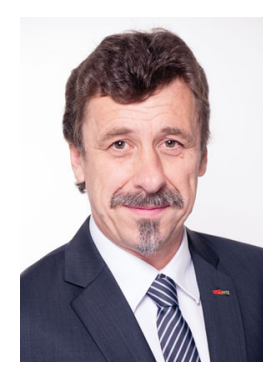

\section{Herwig Renner}

holds a position as associate professor at Graz University of Technology. His research is in the field of power system planning and operation with special focus on transmission system dynamics and industrial power quality. 